\title{
Slip dynamics at an interface between dissimilar materials
}

\author{
K. Ranjith *, J.R. Rice \\ Division of Engineering and Applied Sciences and Department of Earth and Planetary Sciences, \\ Harvard University, Cambridge, MA 02138, USA
}

Received 10 December 1999; received in revised form 28 March 2000

\begin{abstract}
It has been shown recently that steady frictional sliding along an interface between dissimilar elastic solids with Coulomb friction acting at the interface is ill-posed for a wide range of material parameters and friction coefficients. The ill-posedness is manifest in the unstable growth of interfacial disturbances of all wavelengths, with growth rate inversely proportional to the wavelength. We first establish the connection between the ill-posedness and the existence of a certain interfacial wave in frictionless contact, called the generalized Rayleigh wave. Precisely, it is shown that for material combinations where the generalized Rayleigh wave exists, steady sliding with Coulomb friction is ill-posed for arbitrarily small values of friction. In addition, intersonic unstable modes and supersonic steady-state modes exist for sufficiently large values of the friction coefficient. Secondly, regularization of the problem by an experimentally motivated friction law is studied. We show that a friction law with no instantaneous dependence on normal stress but a simple fading memory of prior history of normal stress makes the problem well-posed. (C) 2001 Published by Elsevier Science Ltd.
\end{abstract}

Keywords: A. Dynamics; B. Friction; B. Elastic material; B. Stress waves; B. Stability and well-posedness

\section{Introduction}

Recent work (Renardy (1992), Adams (1995), Martins et al. (1995), Martins and Simões (1995) and Simões and Martins (1998)) has shown the ill-posedness, in the sense described below, of steady sliding of an elastic half-space against a dissimilar

* Corresponding author. Fax: +1-617-495-9837.

E-mail address: ranjith@esag.harvard.edu (K. Ranjith). 
elastic half-space when Coulomb friction acts at their interface. Let $V$ denote the velocity of steady sliding, the same at every point along the interface, and $\tau$ the shear stress at the interface. When the shear stress is perturbed in a single spatial mode of wavenumber $k$,

$$
\Delta \tau=Q(t) \mathrm{e}^{i k x_{1}},
$$

where $x_{1}$ is the coordinate axis along the interface and $Q(t)$ is an arbitrary function of time, $t$, propagating slip-rate modes of form

$$
\Delta V=A(k) \mathrm{e}^{i k\left(x_{1}-c t\right)} \mathrm{e}^{a|k| t}
$$

are found, $A(k)$ is the amplitude of the mode, $a$ and $c$ are independent of the wavelength, and where $a>0$ for a broad range of friction coefficients and material pairs. For such $a>0$ cases, all wavelengths in the slip response are unstable and the growth rate of the instability is inversely proportional to the wavelength. An observer traveling with the phase velocity $c$ of the instability sees a perturbation velocity field that is the sum of an infinite number of modes, namely,

$$
\Delta V(x+c t, t)=\int_{-\infty}^{+\infty} A(k) \mathrm{e}^{i k x} \mathrm{e}^{a|k| t} \mathrm{~d} k
$$

where $x=x_{1}-c t$. Clearly, this integral fails to exist (diverges by oscillation for $x \neq 0$ ) in an arbitrarily small time after the perturbation is turned on, unless $A(k)$ decays exponentially or faster with $|k|$. Such a problem is said to be ill-posed.

Renardy (1992) studied the sliding of a neo-Hookean elastic solid against a rigid substrate. In the limit of linear elasticity, he showed that ill-posedness exists when the friction coefficient is sufficiently high, namely, greater than unity. This limiting case was studied independently by Martins et al. (1995). On the other hand, Adams (1995) showed that when the two solids on either side of the interface are linear elastic and not very dissimilar, the problem can be ill-posed for arbitrarily small values of friction.

Earlier, Weertman (1963) and Achenbach and Epstein (1967) had shown that in frictionless sliding of dissimilar elastic half-spaces, constrained against formation of opening gaps, an interfacial wave solution can exist when the material mismatch is not very high. It is called the generalized Rayleigh wave since its speed of propagation reduces to that of the Rayleigh surface wave when the two materials are identical. The numerical results of Adams (1995) suggested a connection between the existence of the generalized Rayleigh wave and the ill-posedness. fThis is fully explored in our present work. We show that for conditions under which the generalized Rayleigh wave exists in frictionless contact, the stability problem with Coulomb friction is ill-posed for arbitrarily small values of friction.

Weertman (1980) argued that when such a wave exists, a self-healing slip pulse can propagate along the frictional interface between dissimilar elastic solids, even when the remote shear stress is less than the frictional strength of the interface, and a family of such pulse solutions has been constructed by Adams (1998). The velocity 
of propagation of the slip pulse is precisely that of the generalized Rayleigh wave. Numerical studies of the nucleation and propagation of such slip pulses with a Coulomb friction law at the interface, by Andrews and Ben-Zion (1997), Ben-Zion and Andrews (1998) and Harris and Day (1997) have difficulties that seem to have their origin in the instability and ill-posedness results cited earlier. They observe that their simulations depend on mesh size and that a nucleated slip pulse splits into a number of pulses. Using the spectral numerical methodology for bi-materials (Breitenfeld and Geubelle (1998)), Cochard and Rice (2001) illustrate the ill-posedness by showing that the more terms in their spectral basis set, the more the pulse splitting for a case which is ill-posed in the sense discussed above. They show that the same method gives results which converge with enlargement of the basis set for parameter choices in the well-posed range.

We show that an experimentally motivated friction law incorporating a memory dependence, rather than instantaneous dependence, on normal stress regularizes the problem of steady sliding along an interface between dissimilar elastic solids. A friction law for this problem must necessarily incorporate the response to varying normal stress, since slip along a dissimilar material interface alters normal stress. Creep-slippage experiments by Linker and Dieterich (1992) at sliding rates of order $1 \mu \mathrm{m} / \mathrm{s}$ suggest that friction has an instantaneous as well as a memory dependence on normal stress. That is, in response to a step change $\Delta \sigma$ in compressive normal stress at approximately constant slip rate, which will ultimately lead to shear strength increase of $f \Delta \sigma$, a partial strength increase of $(f-\alpha) \Delta \sigma$ occurs at the time of the step and a further memory-like increase $\alpha \Delta \sigma$ occurs with continuing slip over a few $\mu \mathrm{m}$ distance. They found $\alpha \approx(0.3$ to 0.8$) f$, depending on how their data was fit. But subsequent work by Prakash and Clifton (1993) and Prakash (1998) on high-speed sliding (1 to $10 \mathrm{~m} / \mathrm{s})$ induced by oblique shock impact, in which normal stress was altered over a much shorter time scale by a wave reflection, showed no instantaneous effect, but just a fading memory of prior history of normal stress (i.e., $\alpha=f$ ). In this work, we use a friction law suggested by the latter experiments. Previous studies by Martins and Simões (1995) and Simões and Martins (1998) show that regularization can also be achieved by using a friction law in which the usual instantaneous dependence on normal stress is replaced by a dependence on normal stress averaged over some small finite area, although such a law seems not to be directly motivated by experiments and would not, for example, be consistent with memory effects in those just mentioned, for which normal stress was altered uniformly over a macroscopic sliding surface.

This paper is organized as follows: In Section 2, the governing elastodynamic relations between perturbations (from steady sliding) in slip and opening and those in shear and normal stress at the interface are derived. These relations are used in subsequent sections to derive the governing equations for stability. First, the case of frictionless sliding is reviewed in Section 3, leading to a discussion of the generalized Rayleigh wave of Weertman (1963) and Achenbach and Epstein (1967). In Section 4, slip stability with Coulomb friction at the interface is studied. A simpler rederivation of the ill-posedness results of Adams (1995) is presented. The connection between the existence of the generalized Rayleigh wave and the ill-posedness is 
explicity shown. In Section 5, regularization of the problem with a simple, experimentally motivated friction law incorporating the effect of normal stress changes is discussed. The results are finally summarized in Section 6.

\section{Elastodynamic relations}

Let $\left(x_{1}, x_{2}\right)$ be Cartesian coordinates defined such that the slip plane lies at $x_{2}=0$ (see Fig. 1) and the steady state velocities of the material points on either side of the interface, $x_{2}=0^{+}$and $x_{2}=0^{-}$are, respectively, $+V / 2$ and $-V / 2$. Far away from the interface, a shear stress $\tau_{\infty}$ equal to the frictional strength of the interface is applied, i.e. $\tau_{\infty}=f \sigma_{\infty}$, where $f$ is the Coulomb friction coefficient and $\sigma_{\infty}$ is the remote compressive normal stress. The shear and dilatational wave speeds of the material in the region $x_{2} \geq 0^{+}$are denoted by $c_{\mathrm{s} 1}$ and $c_{\mathrm{d} 1}$, respectively. Corresponding wave speeds in the lower half space, $x_{2} \leq 0^{-}$are denoted by $c_{\mathrm{s} 2}$ and $c_{\mathrm{d} 2}$, respectively. Similar notation is used for the densities, $\rho_{1}$ and $\rho_{2}$, shear moduli, $\mu_{1}$ and $\mu_{2}$ and Poisson's ratios, $v_{1}$ and $v_{2}$, of the two solids. Without loss of generality, we assume that $c_{\mathrm{s} 2}>c_{\mathrm{s} 1}$.

Let $\sigma_{\alpha \beta}\left(x_{1}, x_{2}, t\right)$ and $u_{\alpha}\left(x_{1}, x_{2}, t\right)$ denote the stress and displacement fields. In this section, we derive elastodynamic relations between perturbations in traction components of the stress on the interface,

$$
\tau_{\alpha}\left(x_{1}, t\right)=\sigma_{2 \alpha}\left(x_{1}, 0, t\right)
$$

and those in displacement discontinuities,

$$
\delta_{\alpha}\left(x_{1}, t\right)=u_{\alpha}\left(x_{1}, 0^{+}, t\right)-u_{x}\left(x_{1}, 0^{-}, t\right)
$$

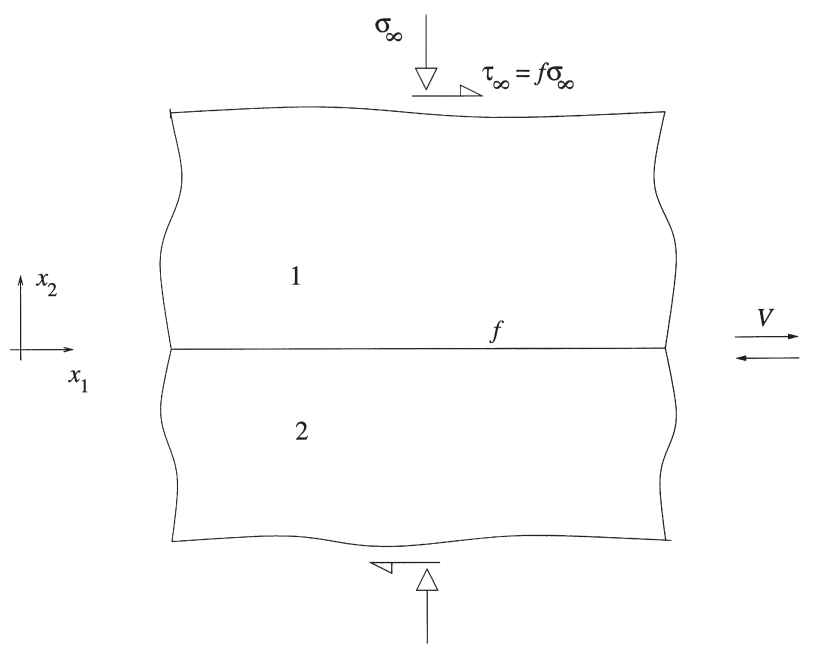

Fig. 1. Frictional sliding along an interface between dissimilar materials. 
at the interface. We restrict our study to the case where $V \ll c_{\mathrm{s} 1}$, which is generally the regime of interest.

Consider a perturbation field in a single spatial mode of the form

$$
\begin{aligned}
& \tau_{1}\left(x_{1}, t\right)=\tau_{\infty}+T_{1}(t) \mathrm{e}^{i k x_{1}}, \\
& \tau_{2}\left(x_{1}, t\right)=-\sigma_{\infty}+T_{2}(t) \mathrm{e}^{i k x_{1}}, \\
& u_{1}^{ \pm}\left(x_{1}, t\right)= \pm V t / 2+U_{1}^{ \pm}(t) \mathrm{e}^{i k x_{1}}, \\
& u_{2}^{ \pm}\left(x_{1}, t\right)=U_{2}^{ \pm}(t) \mathrm{e}^{i k x_{1}} .
\end{aligned}
$$

Denoting the Laplace transform with respect to $t$ by

$$
\hat{g}(p)=\int_{0}^{\infty} \mathrm{e}^{-p t} g(t) \mathrm{d} t,
$$

following Geubelle and Rice (1995), it can be shown that perturbations in displacements at $x_{2}=0^{+}$are related to those in shear and normal stress by

$$
\left\{\begin{array}{c}
\hat{T}_{1} \\
\hat{T}_{2}
\end{array}\right\}=\left[\begin{array}{ll}
\hat{G}_{11} & \hat{G}_{12} \\
\hat{G}_{21} & \hat{G}_{22}
\end{array}\right]\left\{\begin{array}{c}
\hat{U}_{1}^{+} \\
\hat{U}_{2}^{+}
\end{array}\right\}
$$

where

$$
\begin{aligned}
& \hat{G}_{11}(p, k)=-\mu_{1}|k| \frac{\alpha_{\mathrm{d} 1}\left(1-\alpha_{\mathrm{s} 1}^{2}\right)}{1-\alpha_{\mathrm{s} 1} \alpha_{\mathrm{d} 1}}, \\
& \hat{G}_{22}(p, k)=-\mu_{1}|k| \frac{\alpha_{\mathrm{s} 1}\left(1-\alpha_{\mathrm{s} 1}^{2}\right)}{1-\alpha_{\mathrm{s} 1} \alpha_{\mathrm{d} 1}}, \\
& \hat{G}_{12}(p, k)=-i \mu_{1} k\left(2-\frac{1-\alpha_{\mathrm{s} 1}^{2}}{1-\alpha_{\mathrm{s} 1} \alpha_{\mathrm{d} 1}}\right)=-\hat{G}_{21}(p, k),
\end{aligned}
$$

with

$$
\alpha_{\mathrm{s} 1}=\sqrt{1+s^{2} / c_{\mathrm{s} 1}^{2}}, \quad \alpha_{\mathrm{d} 1}=\sqrt{1+s^{2} / c_{\mathrm{d} 1}^{2}} \text { and } s=p /|k| .
$$

To ensure bounded displacements in $x_{2} \geq 0$, (see Geubelle and Rice (1995), eq. 14), we require that the real parts of $\alpha_{\mathrm{s} 1}$ and $\alpha_{\mathrm{d} 1}$ be non-negative in the physical domain $\operatorname{Re}(s) \geq 0$. We make the real parts non-negative for all $s$ by defining branch cuts in the complex $s$-plane along $\left(-i \infty,-i c_{\mathrm{s} 1}\right]$ and $\left[i c_{\mathrm{s} 1},+i \infty\right)$ for $\alpha_{\mathrm{s} 1}$ and, similarly, along $\left(-i \infty,-i c_{\mathrm{d} 1}\right]$ and $\left[+i c_{\mathrm{d} 1},+i \infty\right)$ for $\alpha_{\mathrm{d} 1}$.

Inverting Eq. (6) we get

$$
\left\{\begin{array}{c}
\hat{U}_{1}^{+} \\
\hat{U}_{2}^{+}
\end{array}\right\}=\left[\begin{array}{ll}
\hat{C}_{11}^{+} & \hat{C}_{12}^{+} \\
\hat{C}_{21}^{+} & \hat{C}_{22}^{+}
\end{array}\right]\left\{\begin{array}{l}
\hat{T}_{1} \\
\hat{T}_{2}
\end{array}\right\}
$$


where

$$
\begin{aligned}
& \hat{C}_{11}^{+}(p, k)=-\frac{1}{\mu_{1}|k|} \frac{\alpha_{\mathrm{s} 1}\left(1-\alpha_{\mathrm{s} 1}^{2}\right)}{R_{1}(s)}, \\
& \hat{C}_{22}^{+}(p, k)=-\frac{1}{\mu_{1}|k|} \frac{\alpha_{\mathrm{d} 1}\left(1-\alpha_{\mathrm{s} 1}^{2}\right)}{R_{1}(s)}, \\
& \hat{C}_{12}^{+}(p, k)=-\frac{1}{i \mu_{1} k} \frac{2 \alpha_{\mathrm{s} 1} \alpha_{\mathrm{d} 1}-\left(1+\alpha_{\mathrm{s} 1}^{2}\right)}{R_{1}(s)}=-\hat{C}_{21}^{+}(p, k),
\end{aligned}
$$

with

$$
R_{1}(s)=4 \alpha_{\mathrm{s} 1} \alpha_{\mathrm{d} 1}-\left(1+\alpha_{\mathrm{s} 1}^{2}\right)^{2} .
$$

The properties of the Rayleigh function $R_{1}(s)$ are discussed, for instance, in Achenbach (1973). The function has a double zero at $s=0$ and simple zeros at $s= \pm i c_{\mathrm{R} 1}$, where $c_{\mathrm{R} 1}$ is the speed of propagation of the Rayleigh wave at a free surface of the material. It is important to note that the combination

$$
\hat{C}_{11}^{+} \hat{C}_{22}^{+}-\hat{C}_{12}^{+} \hat{C}_{21}^{+}=\left(1-\alpha_{\mathrm{s} 1} \alpha_{\mathrm{d} 1}\right) / \mu_{1}^{2} k^{2} R_{1}(s),
$$

and thus has simple poles at $s= \pm i c_{\mathrm{R} 1}$, and not double poles as may be naively expected.

Analogous equations at $x_{2}=0^{-}$are

$$
\left\{\begin{array}{c}
\hat{U}_{1}^{-} \\
\hat{U}_{2}^{-}
\end{array}\right\}=\left[\begin{array}{ll}
-\hat{C}_{11}^{-} & \hat{C}_{12}^{-} \\
\hat{C}_{21}^{-} & -\hat{C}_{22}^{-}
\end{array}\right]\left\{\begin{array}{c}
\hat{T}_{1} \\
\hat{T}_{2}
\end{array}\right\}
$$

where

$$
\begin{aligned}
& \hat{C}_{11}^{-}(p, k)=-\frac{1}{\mu_{2}|k|} \frac{\alpha_{\mathrm{s} 2}\left(1-\alpha_{\mathrm{s} 2}^{2}\right)}{R_{2}(s)}, \\
& \hat{C}_{22}^{-}(p, k)=-\frac{1}{\mu_{2}|k|} \frac{\alpha_{\mathrm{d} 2}\left(1-\alpha_{\mathrm{s} 2}^{2}\right)}{R_{2}(s)}, \\
& \hat{C}_{12}^{-}(p, k)=-\frac{1}{i \mu_{2} k} \frac{2 \alpha_{\mathrm{s} 2} \alpha_{\mathrm{d} 2}-\left(1+\alpha_{\mathrm{s} 2}^{2}\right)}{R_{2}(s)}=-\hat{C}_{21}^{-}(p, k),
\end{aligned}
$$

with

$$
\alpha_{\mathrm{s} 2}=\sqrt{1+s^{2} / c_{\mathrm{s} 2}^{2}}, \alpha_{\mathrm{d} 2}=\sqrt{1+s^{2} / c_{\mathrm{d} 2}^{2}} \text { and } R_{2}(s)=4 \alpha_{\mathrm{s} 2} \alpha_{\mathrm{d} 2}-\left(1+\alpha_{\mathrm{s} 2}^{2}\right)^{2} .
$$

Branch cuts for $\alpha_{\mathrm{s} 2}$ and $\alpha_{\mathrm{d} 2}$ are defined analogous to those for $\alpha_{\mathrm{s} 1}$ and $\alpha_{\mathrm{d} 1}$ to ensure bounded displacements in the half space $x_{2} \leq 0$. The non-trivial roots of the Rayleigh function $R_{2}(s)$ are denoted by $s= \pm i c_{\mathrm{R} 2}$. Note that the combination $\left(\hat{C}_{11}^{-} \hat{C}_{22}^{-}-\hat{C}_{12}^{-} \hat{C}_{21}^{-}\right)$ only has simple poles at $s= \pm i c_{\mathrm{R} 2}$.

Writing the perturbed slip and opening as 


$$
\begin{aligned}
& \delta_{1}\left(x_{1}, t\right)=V t+D_{1}(t) \mathrm{e}^{i k x_{1}}, \\
& \delta_{2}\left(x_{1}, t\right)=D_{2}(t) \mathrm{e}^{i k x_{1}},
\end{aligned}
$$

we can subtract Eq. (13) from Eq. (9) at low slip rates, $V \ll c_{\mathrm{s} 1}$, to get

$$
\left\{\begin{array}{l}
\hat{D}_{1} \\
\hat{D}_{2}
\end{array}\right\}=\left[\begin{array}{ll}
\hat{K}_{11} & \hat{K}_{12} \\
\hat{K}_{21} & \hat{K}_{22}
\end{array}\right]\left\{\begin{array}{l}
\hat{T}_{1} \\
\hat{T}_{2}
\end{array}\right\}
$$

where

$$
\hat{K}_{11}=\hat{C}_{11}^{+}+\hat{C}_{11}^{-}, \hat{K}_{22}=\hat{C}_{22}^{+}+\hat{C}_{22}^{-}, \hat{K}_{12}=\hat{C}_{12}^{+}-\hat{C}_{12}^{-}=-\hat{K}_{21} .
$$

The functions $\hat{K}_{i j}$ have simple poles at $s= \pm i c_{\mathrm{R} 1}$ and at $s= \pm i c_{\mathrm{R} 2}$.

We will find it convenient to use the inverse of Eq. (17), namely

$$
\left\{\begin{array}{l}
\hat{T}_{1} \\
\hat{T}_{2}
\end{array}\right\}=\left[\begin{array}{ll}
\hat{M}_{11} & \hat{M}_{12} \\
\hat{M}_{21} & \hat{M}_{22}
\end{array}\right]\left\{\begin{array}{l}
\hat{D}_{1} \\
\hat{D}_{2}
\end{array}\right\}
$$

where

$$
\hat{M}_{11}=\hat{K}_{22} / D, \hat{M}_{22}=\hat{K}_{11} / D, \hat{M}_{12}=-\hat{K}_{12} / D=-\hat{M}_{21},
$$

with

$$
D=\hat{K}_{11} \hat{K}_{22}-\hat{K}_{12} \hat{K}_{21}
$$

The combination $\left(\hat{K}_{11} \hat{K}_{22}-\hat{K}_{12} \hat{K}_{21}\right)$ only has simple poles at $s= \pm i c_{\mathrm{R} 1}$ and $s= \pm i c_{\mathrm{R} 2}$, following our previous discussion of the poles of a similar combination of $\hat{C}_{i j}^{+}$. Thus, the Rayleigh poles in the numerators of the expressions for $\hat{M}_{i j}$ cancel those in the denominator.

Suppose that a perturbation in shear stress is applied at the sliding interface (e.g. due to a wave incident on the interface) of the form

$$
\Delta \tau_{1}(t, k)=Q(t) \mathrm{e}^{i k x_{1}}
$$

such that $Q(t)=0$ for $t<0$ and $Q(t)$ is arbitrary for $t>0$. We interpret this as an additional stress (to $\tau_{\infty}$ ) that would have been supported if the interface was constrained against further slip (than $V t$ ) or opening. The total change in shear and normal stresses at the interface is the sum of externally applied perturbation and those due to heterogeneous slip and opening, given by Eq. (19):

$$
\left\{\begin{array}{l}
\hat{T}_{1} \\
\hat{T}_{2}
\end{array}\right\}=\left[\begin{array}{ll}
\hat{M}_{11} & \hat{M}_{12} \\
\hat{M}_{21} & \hat{M}_{22}
\end{array}\right]\left\{\begin{array}{l}
\hat{D}_{1} \\
\hat{D}_{2}
\end{array}\right\}+\left\{\begin{array}{l}
\hat{Q} \\
0
\end{array}\right\}
$$

where $\hat{Q}(p)$ is the Laplace transform of $Q(t)$. In the subsequent sections, we determine the sliding response $\hat{D}_{1}$ due to the applied $\hat{Q}$, when various friction laws are operative at the interface. 


\section{Frictionless sliding: the generalized Rayleigh wave}

First, consider the case when there is no friction at the interface. Setting $\hat{T}_{1}=0$ and $\hat{D}_{2}=0$ in Eq. (22) and using Eq. (20), we get

$$
\hat{D}_{1}=-\frac{\hat{K}_{11} \hat{K}_{22}-\hat{K}_{12} \hat{K}_{21}}{\hat{K}_{22}} \hat{Q} \text {. }
$$

As noted earlier, the Rayleigh poles in the denominator and numerator of the transfer function cancel each other. The only poles of the transfer function are the roots of the equation

$$
\hat{K}_{22}(s)=0 \text {, }
$$

which is precisely the equation for the generalized Rayleigh wave of Weertman (1963) and Achenbach and Epstein (1967). Cancelling the Rayleigh poles in the numerator and denominator of the transfer function, the above equation can be written in the form

$$
-\hat{K}_{22}(s) R_{1}(s) R_{2}(s) \mu_{1} c_{\mathrm{s} 2}^{2}|k| / s^{2}=\left(\mu_{1} / \mu_{2}\right) \alpha_{\mathrm{d} 2}(s) R_{1}(s)+\left(c_{\mathrm{s} 2} / c_{\mathrm{s} 1}\right)^{2} \alpha_{\mathrm{d} 1}(s) R_{2}(s)=0 .
$$

Weertman (1963) and Achenbach and Epstein (1967) showed that when the two materials are not very dissimilar, the above equation has pure imaginary roots $s_{\mathrm{o}}= \pm i c_{\mathrm{GR}}$ corresponding to steady interfacial wave propagation. When $c_{\mathrm{R} 2}<c_{\mathrm{s} 1}$, such roots always exist and the speed of the wave $c_{\mathrm{GR}}$ is such that

$$
\min \left(c_{\mathrm{R} 1}, c_{\mathrm{R} 2}\right)<c_{\mathrm{GR}}<\max \left(c_{\mathrm{R} 1}, c_{\mathrm{R} 2}\right) .
$$

On the other hand, when $c_{\mathrm{R} 2}>c_{\mathrm{s} 1}$, roots exist when

$$
\rho_{2} / \rho_{1}<\left(c_{\mathrm{s} 1} / c_{\mathrm{s} 2}\right)^{4} \alpha_{\mathrm{d} 2}\left(i c_{\mathrm{s} 1}\right) / \alpha_{\mathrm{d} 1}\left(i c_{\mathrm{s} 1}\right) R_{2}\left(i c_{\mathrm{s} 1}\right)
$$

and the wave speed $c_{\mathrm{GR}}<c_{\mathrm{s} 1}$. For modestly different densities and Poisson's ratios, the generalized Rayleigh wave exists for mismatches in shear wave speeds up to about $40 \%$.

\section{Ill-posedness with Coulomb friction}

In this section, a simpler rederivation of the ill-posedness results of Adams (1995) and Martins et al. (1995) is first presented. This is then used to establish the relationship between the ill-posedness and the existence of the generalized Rayleigh wave.

Taking $V>0$, the Coulomb friction law is

$$
\tau_{1}=-f \tau_{2}
$$


As before, we study the stability of sliding response to perturbations from the steady state at velocity $V$. Solving for $\hat{D}_{1}$ in Eq. (22) with the constraints

$$
\hat{T}_{1}=-f \hat{T}_{2}, \hat{D}_{2}=0
$$

we get,

$$
\hat{D}_{1}=-\frac{\hat{K}_{11} \hat{K}_{22}-\hat{K}_{12} \hat{K}_{21}}{\hat{K}_{22}-f \hat{K}_{21}} \hat{Q} .
$$

The poles in the numerator of the above expression corresponding to the Rayleigh waves of the two materials are again cancelled by identical poles in the denominator. For instability, we thus need that a root of the equation

$$
\hat{K}_{22}(s)-f \hat{K}_{21}(s)=0
$$

have a positive real part. Recalling from Eq. (18), Eq. (10) and Eq. (14) that $\hat{K}_{22}$ has $1 /|k|$ dependence on $k$ and that $\hat{K}_{21}$ is proportional to $1 / k$, the roots of the above equation depend only on the sign of $k$ and not its magnitude.

The kernels $\hat{K}_{22}$ and $\hat{K}_{21}$ have the following properties:

$$
\begin{aligned}
& \hat{K}_{22}(s, k)=\hat{K}_{22}(\bar{s}, k)=\hat{K}_{22}(-s, k)=\hat{K}_{22}(s,-k), \\
& \hat{K}_{21}(s, k)=-\hat{K}_{21}(\bar{s}, k)=\hat{K}_{21}(-s, k)=-\hat{K}_{21}(s,-k),
\end{aligned}
$$

where the overbar denotes complex conjugation. We see immediately that if $s^{*}$ is a root of Eq. (28), so is $-s^{*}$. Also, if $s^{*}$ and $-s^{*}$ are the roots for wavenumber $k, \overline{s^{*}}$ and $-\overline{s^{*}}$ are the roots for wavenumber $-k$. Writing

$$
\operatorname{Re}\left(s^{*}\right)=a \text { and }-\operatorname{sign}(k) \operatorname{Im}\left(s^{*}\right)=c,
$$

the slip response to perturbations with wavenumber $k$ or $-k$ has propagating modes of the form

$$
\delta_{1}\left(x_{1}, t\right) \sim\left(\mathrm{e}^{i k\left(x_{1}-c t\right)} \mathrm{e}^{+a|k| t}, \mathrm{e}^{i k\left(x_{1}+c t\right)} \mathrm{e}^{-a|k| t}, \mathrm{e}^{-i k\left(x_{1}-c t\right)} \mathrm{e}^{+a|k| t}, \mathrm{e}^{-i k\left(x_{1}+c t\right)} \mathrm{e}^{-a|k| t}\right) .
$$

The modes propagate in opposite directions with phase velocity $c$. When a root of Eq. (26) has a positive real part $a$, one mode grows with an exponent of $a|k|$, while the other decays at the same rate. Thus there is unstable growth in the modal response with the growth rate being faster for short wavelengths. Further, the growing modes associated with a given root propagate with a unique velocity $c$ and in the same direction along the interface for $k$ and $-k$. When $c>0$, the unstable modes propagate in the positive $x_{1}$ direction and when $c<0$, they travel in the negative $x_{1}$ direction. Note also from Eq. (29) and Eq. (30) that when a root exists with a real part $a$, then a root also exists with that same real part $a$ when $f$ is changed to $-f$.

\subsection{Ill-posedness at arbitrarily small $f$ when $c_{G R}$ exists}

Here, we prove that when the material properties on either side of the interface are such that a generalized Rayleigh wave exists in frictionless sliding, the stability problem with Coulomb friction is ill-posed for arbitrarily small values of the friction 
coefficient. In Section 3, we saw that when the generalized Rayleigh wave exists, the equation $\hat{K}_{22}(s)=0$ has two imaginary roots, $s_{\mathrm{o}}= \pm i c_{\mathrm{GR}}$. We now show using a perturbation analysis that when $f$ is changed from zero, one root moves into the right half $s$-plane while the other moves into the left half plane. The numerical results presented in Section 4.2 are in agreement with this analysis.

For small values of $f$, we expect roots of $\hat{K}_{22}-f \hat{K}_{21}$ close to $s_{\mathrm{o}}$. Using a perturbation expansion of the form

$$
s=s_{\mathrm{o}}+f s_{1}+\cdots
$$

for the roots in Eq. (28) and retaining $O(f)$ terms, we get

$$
s_{1}=\hat{K}_{21}\left(s_{\mathrm{o}}\right) / \hat{K}_{22}^{\prime}\left(s_{o}\right) \text {. }
$$

Since $\hat{K}_{21}\left(s_{\mathrm{o}}\right)$ and $\hat{K}_{22}^{\prime}\left(s_{o}\right)$ are purely imaginary, $s_{1}$ is a real number. Furthermore, it follows from Eq. (29) and Eq. (30) that

$$
\begin{aligned}
& \hat{K}_{21}\left( \pm i c_{\mathrm{GR}}\right)=\hat{K}_{21}\left(+i c_{\mathrm{GR}}\right), \\
& \hat{K}_{22}^{\prime}\left( \pm i c_{\mathrm{GR}}\right)= \pm \hat{K}_{22}^{\prime}\left(+i c_{\mathrm{GR}}\right) .
\end{aligned}
$$

Therefore, the term $f_{s_{1}}$ in the perturbation expansion is of opposite signs (and real) for the two values $s_{\mathrm{O}}= \pm i c_{\mathrm{GR}}$. The roots move from $s_{\mathrm{o}}= \pm i c_{\mathrm{GR}}$ parallel to the $\operatorname{Re}(s)$ axis as $f$ is changed from zero. One root moves into the right half $s$-plane, while the other moves to the left. This makes the problem ill-posed for arbitrarily small friction for cases where the generalized Rayleigh wave exists in frictionless contact. Note in particular that the argument would apply for positive or negative $f$, both giving ill-posedness.

Suppose now that the two solids are sufficiently different such that the generalized Rayleigh wave does not exist in frictionless contact. This means that the equation $\hat{K}_{22}(s)=0$ has no roots, not only along the imaginary axis but in the whole complex plane. The latter is easily seen as follows. From the discussion of roots given in the previous subsection, if there exists any root with non-zero $\operatorname{Re}(s)$, in the response to an $\mathrm{e}^{i k x_{1}}$ perturbation, then there must exist a root with $\operatorname{Re}(s)>0$, hence showing growth in time. But this violates energy conservation, which must apply in the frictionless case, as argued below. The two sliding bodies have a strain energy corresponding to uniform stressing in the unperturbed configuration, and have a kinetic energy corresponding to the rigid translation at rate $V$. An $\mathrm{e}^{i k x_{1}}$ perturbation field then provides a positive definite change in both strain energy and kinetic energy (the cross terms of the respective quadratic forms for strain and kinetic energy density integrate to zero). But such energy per wavelength cannot be greater (or smaller) than the energy put into the initial $\mathrm{e}^{i k x_{1}}$ perturbation. Hence no root to $\hat{K}_{22}(s)=0$ with $\operatorname{Re}(s) \neq 0$ can exist. (The same energy conservation argument does not apply, of course, when $f \neq 0$.)

Thus, if no generalized Rayleigh wave exists, $\hat{K}_{22}(s)=0$ has no roots. If we now consider solutions to the problem with friction, $\hat{K}_{22}(s)-f \hat{K}_{21}(s)=0$, it is clear that for arbitrarily small but non-zero $f$, there will be no roots. (Indeed, there will be none for sufficiently small positive or negative $f$.) The possible exception is that a root might emerge at $\infty$ as $f$ is altered slightly from zero, but this possibility is precluded 
by the expressions in Eq. (10) and Eq. (14) which show that $\hat{C}_{21}^{ \pm}(s) / \hat{C}_{22}^{+}(s)$, and hence $\hat{K}_{21}(s) / \hat{K}_{22}(s)$, approach zero as $s \rightarrow \infty$. Thus, when no generalized Rayleigh wave exists, there will be an interval around $f=0$, say $-f_{\mathrm{c}}<f<f_{\mathrm{c}}$ (where $f_{\mathrm{c}}>0$ ) for which there is stable response to an $\mathrm{e}^{i k x_{1}}$ perturbation. Note in particular that the analysis for this case predicts stability even in a range of negative friction coefficients, $-f_{\mathrm{c}}<f<0$.

\subsection{Discussion of roots of $\hat{K}_{22}-f \hat{K}_{21}=0$}

First, we show that for sufficiently large values of $f$, a family of steady-state (i.e., non-growing) supersonic interfacial wave solutions exist. Along the imaginary axis $s=i \zeta$, for $|\zeta| \geq \max \left(c_{\mathrm{d} 1}, c_{\mathrm{d} 2}\right)$, both $\hat{K}_{22}(s)$ and $\hat{K}_{21}(s)$ are pure imaginary. Hence, $f=$ $\hat{K}_{22} / \hat{K}_{21}$ is a real number and $s=i \zeta$ is a root corresponding to that value of $f$. These roots correspond to supersonic waves at the interface. They generalize the ones found by Adams (2000) for a bi-material system with a rigid substrate. Adams' results can be obtained by letting $c_{\mathrm{s} 2} / c_{\mathrm{s} 1} \rightarrow \infty$ and $\mu_{1} / \mu_{2} \rightarrow 0$ in the expressions Eq. (18) for $\hat{K}_{22}(s)$ and $\hat{K}_{21}(s)$ and using them in Eq. (28). This gives

$$
f=\frac{i k}{|k|} \frac{\alpha_{\mathrm{d} 1}\left(1-\alpha_{\mathrm{s} 1}^{2}\right)}{2 \alpha_{\mathrm{s} 1} \alpha_{\mathrm{d} 1}-\left(1+\alpha_{\mathrm{s} 1}^{2}\right)} .
$$

Clearly, the right hand side is a real number when $s=i \zeta$ is purely imaginary with $|\zeta| \geq c_{\mathrm{d} 1}$.

To numerically compute the roots of Eq. (28) in the complex s-plane, we use the property that since $f$ is a real number, $\operatorname{Im}\left(\hat{K}_{22} / \hat{K}_{21}\right)=0$ at any root location. The following steps are performed:

1. Fix a value of $\operatorname{Re}(s)=r$.

2. Determine all values of $s$ with $\operatorname{Re}(s)=r$ for which $\operatorname{Im}\left(\hat{K}_{22} / \hat{K}_{21}\right)=0$.

3. Compute $f=\hat{K}_{22} / \hat{K}_{21}$. Ignore the root if $f<0$.

The case studied in Figs. 2 and 3 is one where there is a modest mismatch in material properties across the interface such that the generalized Rayleigh wave exists in frictionless contact. The material properties are $c_{\mathrm{s} 2} / c_{\mathrm{s} 1}=1.2, \rho_{2} / \rho_{1}=1.2, v_{1}=v_{2}=0.25$. For this pair, the speed of the generalized Rayleigh wave is $c_{\mathrm{GR}}=0.9898 c_{\mathrm{s} 1}$. We focus only on the roots in the right half $s$-plane since they determine stability. When $f=0$, there are two roots at $s_{\mathrm{o}}= \pm i c_{\mathrm{GR}}$. As $f$ is increased from zero, one of these roots moves into the right half $s$-plane, as seen in Figs. 2 and 3. Furthermore, Fig. 2 shows that the imaginary part of the root remains approximately constant as $f$ is increased from zero. This implies that the velocity of propagation of the mode is approximately independent of the coefficient of friction in this regime (and thus $c \approx c_{\mathrm{GR}}$, where $c$ is defined as in Eq. (31). These results are consistent with the perturbation analysis presented in the previous section.

For sufficiently high friction, other unstable modes are introduced. At $f=0.22$, two roots appear on the branch cut of $\hat{K}_{22}(s)$ on the imaginary axis. One of these moves 


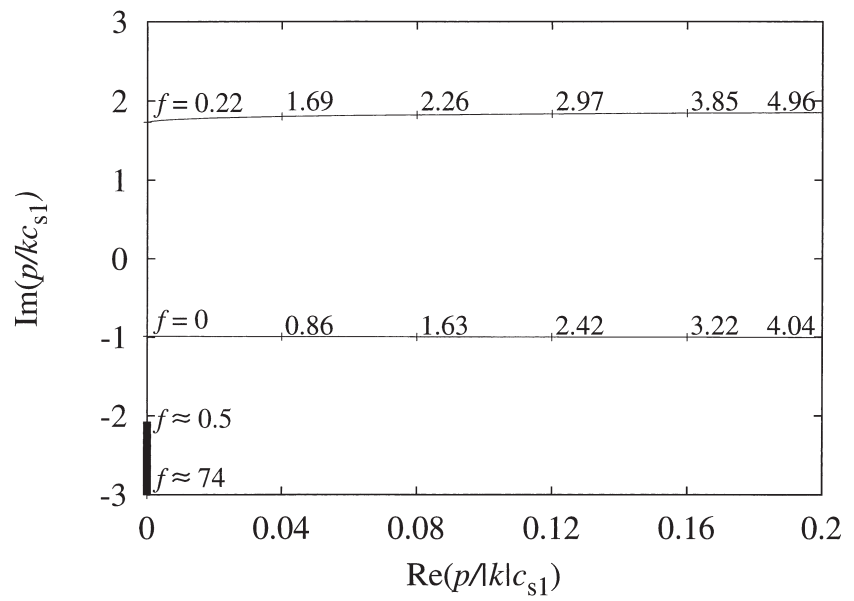

Fig. 2. Root locations of $\hat{K}_{22}(s)-f \hat{K}_{21}(s)=0$ in the complex right half $s$-plane as a function of friction coefficient $f$ for a particular bi-material pair: $c_{\mathrm{s} 2} / c_{\mathrm{s} 1}=1.2, \rho_{2} / \rho_{1}=1.2$, and $v_{1}=v_{2}=0.25$. The generalized Rayleigh wave exists in frictionless contact for this material combination and its speed is $c_{\mathrm{GR}}=0.9898 c_{\mathrm{s} 1}$. Following the analysis in Section 4.1, roots exist in the right half $s$-plane for arbitrarily small $f$.

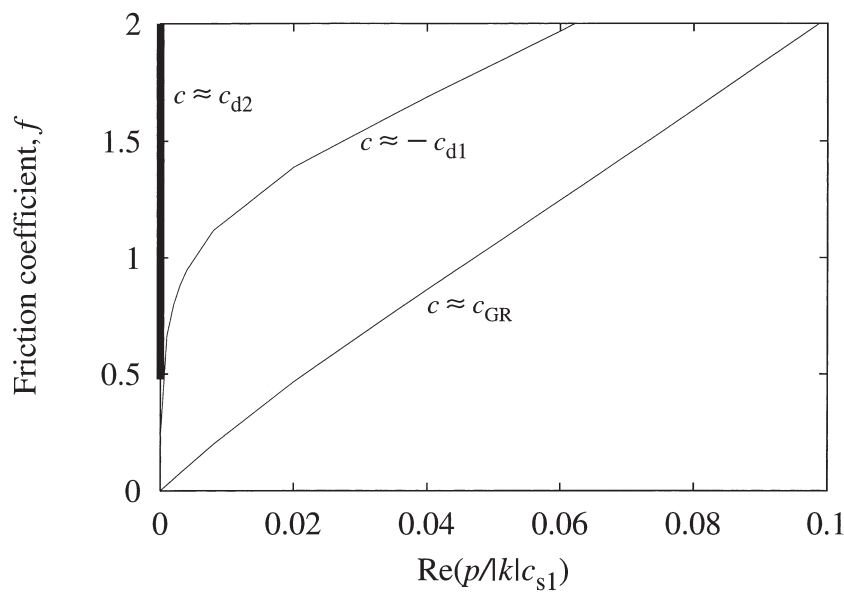

Fig. 3. Normalized growth rate of instability as a function of friction coefficient for a particular bimaterial pair: $c_{\mathrm{s} 2} / c_{\mathrm{s} 1}=1.2, \rho_{2} / \rho_{1}=1.2$, and $v_{1}=v_{2}=0.25$. The generalized Rayleigh wave exists in frictionless contact for this material combination. Following the analysis of Section 4.1, instability occurs for arbitrarily small $f$. As defined in Eq. (31), $c=-\operatorname{Im}(p / k)$ is the phase velocity of the instability. The speed of supersonic modes is close to $c_{\mathrm{d} 2}$ for the range of friction coefficients shown in the figure.

into the right half $s$-plane as $f$ is further increased. Again, the propagation speed along the root path varies only modestly as $f$ is increased, although for both roots, the rate of growth of the instability increases rapidly with $f$ (Fig. 3). In addition to these unstable modes are the family of steady-state supersonic solutions mentioned 


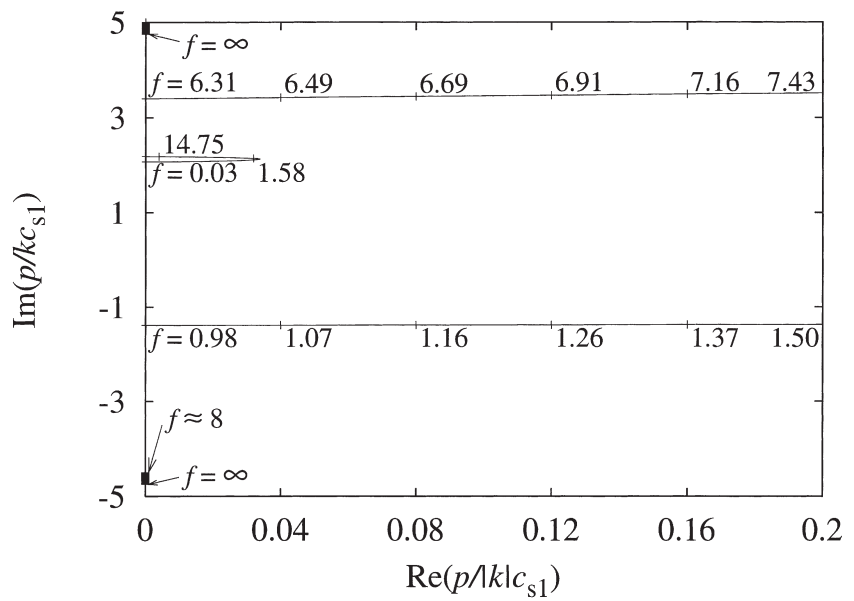

Fig. 4. Root locations of $\hat{K}_{22}(s)-f \hat{K}_{21}(s)=0$ in the complex right half $s$-plane as a function of friction coefficient $f$ for a Steel-PMMA material pair: $c_{\mathrm{s} 2} / c_{\mathrm{s} 1}=2.40, \rho_{2} / \rho_{1}=6.58, v_{1}=0.35$ and $v_{2}=0.3$. The generalized Rayleigh wave does not exist in frictionless contact for this material combination. Following the analysis in Section 4.1, no roots exist in the complex $s$-plane for $|f|<f_{\mathrm{c}}=0.03$.

earlier. They correspond to purely imaginary roots in Figs. 2 and 3 indicated with the thick line.

We next study in Figs. 4 and 5 a material pair with large contrast in properties across the interface so that the generalized Rayleigh wave does not exist in frictionless contact. The properties chosen are for a Steel-PMMA bi-material system:

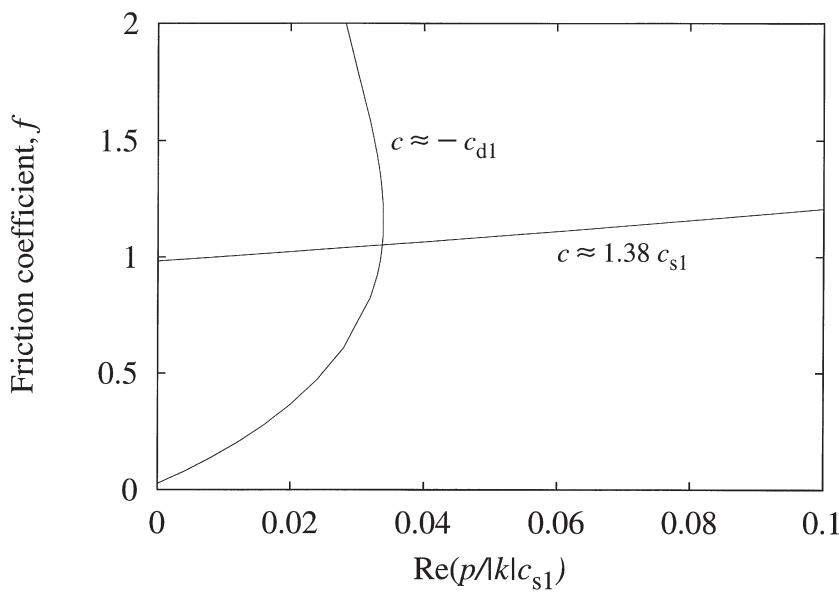

Fig. 5. Normalized growth rate of instability as a function of friction coefficient for a Steel-PMMA material pair: $c_{\mathrm{s} 2} / c_{\mathrm{s} 1}=2.40, \rho_{2} / \rho_{1}=6.58, v_{1}=0.35$ and $v_{2}=0.3$. The generalized Rayleigh wave does not exist in frictionless contact for this material pair. Following the discussion in Section 4.1, instability occurs only when $|f|>f_{\mathrm{c}}=0.03$. As defined in Eq. (31), $c=-\operatorname{Im}(p / k)$ is the phase velocity of the instability. 
$c_{\mathrm{s} 2} / c_{\mathrm{s} 1}=2.40, \rho_{2} / \rho_{1}=6.58, v_{1}=0.35, v_{2}=0.3$. In this case, no roots exist in the right half $s$-plane when $f<0.03$. Hence frictional sliding is stable in this range. At $f=f_{c}=0.03$, a root appears on the right bank of the branch cut of $\hat{K}_{22}(s)-f \hat{K}_{21}(s)$, with propagation speed near $c_{\mathrm{d} 1}$ and moves into the right half $s$-plane as $f$ is further increased. Other unstable modes are also introduced at $f=0.98$ and at the rather uninterestingly large $f=6.31$. In addition, a family of supersonic steady-state solutions also exist at large friction.

Stability diagrams showing the range of values for which the stability problem is ill-posed can be constructed by calculating the value of the friction coefficient $f_{\mathrm{c}}$ at which an unstable right half pole first appears for a particular material combination. Two such diagrams are shown in Figs. 6 and 7, as a function of the shear wave speed mismatch. Fig. 6 is for the case when there is a modest $20 \%$ mismatch in densities between two Poisson materials $\left(v_{1}=v_{2}=0.25\right)$. The figure shows that when the shear wave speeds of the two materials are not too dissimilar so that the generalized Rayleigh wave exists in frictionless contact, $f_{\mathrm{c}}=0$ and the stability problem is ill-posed for arbitrary small values of friction. For slightly larger mismatch in shear wave speeds than in the previous case, an unstable mode propagates in the positive $x_{1}$ direction with a speed in the range (1 to 1.2) $c_{\mathrm{s} 1}$. For still higher values of $c_{\mathrm{s} 2} / c_{\mathrm{s} 1}$, the destabilizing mode propagates at a speed very close to, but slightly lower than $c_{\mathrm{d} 1}$ in the negative $x_{1}$ direction. Another stability diagram is shown in Fig. 7 for material pairs where $\rho_{2} / \rho_{1}=5.0, v_{1}=0.25$ and $v_{2}=0.35$, which are properties typical of a metal/carbon fiber composite bi-material system (approximating the latter as isotropic). Here, as before, $f_{c}=0$ when the shear wave speed mismatch is small enough to allow the existence of the generalized Rayleigh wave. When the generalized Rayleigh wave ceases to exist, an unstable mode propagates with a speed in the range (1 to 1.3$) c_{\mathrm{s} 1}$. For a narrow band of wave speed ratios the first destabiliz-

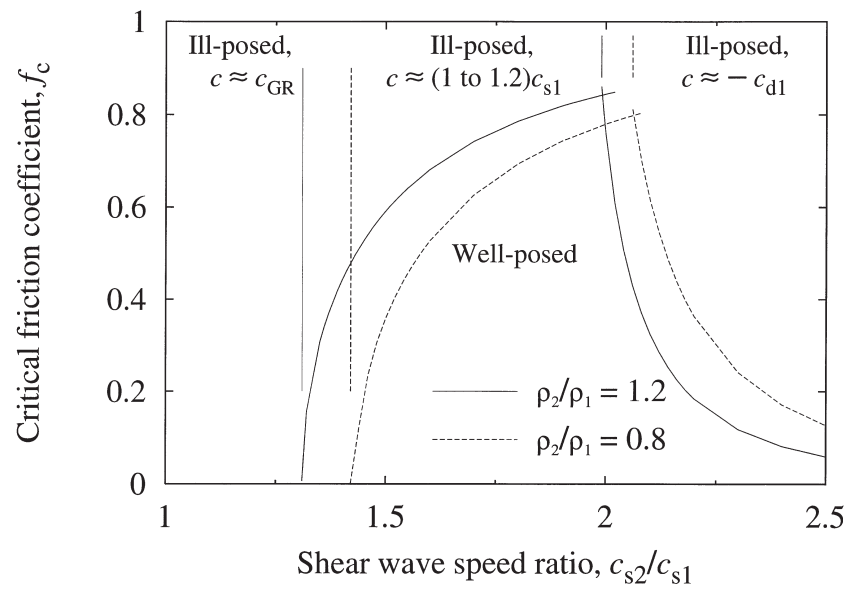

Fig. 6. Stability diagram for two families of bi-material pairs as a function of the shear wave speed ratio. For one pair, the density ratio is $\rho_{2} / \rho_{1}=1.2$ and for the other $\rho_{2} / \rho_{1}=0.8$. For both families of material pairs, $v_{1}=v_{2}=0.25$. As defined in Eq. (31), $c=-\operatorname{Im}(p / k)$ is the phase velocity of the instability. 


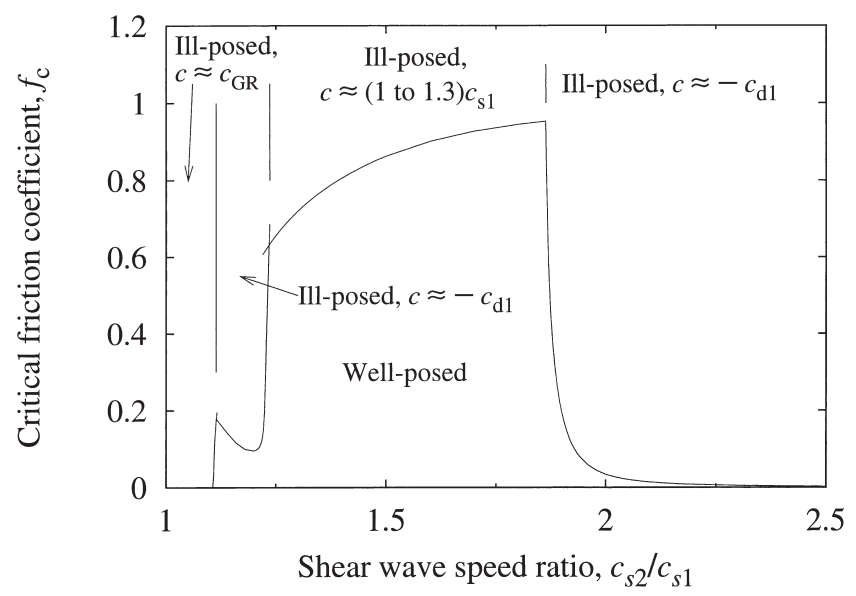

Fig. 7. Stability diagram for a family of bi-material pairs with $\rho_{2} / \rho_{1}=5.0, v_{1}=0.25$ and $v_{2}=0.35$ as a function of the shear wave speed ratio. $c=-\operatorname{Im}(p / k)$, as defined in Eq. (31), is the phase velocity of the instability.

ing mode propagates at a speed close to and slightly higher than $c_{\mathrm{d} 1}$. This mode propagates in the negative $x_{1}$ direction. Again, at large values of $c_{\mathrm{s} 2} / c_{\mathrm{s} 1}$, an unstable mode is introduced at small values of friction that travels with a speed slightly lower than $c_{\mathrm{d} 1}$ in the negative $x_{1}$ direction.

\section{Regularization of steady sliding}

In this section, we study stability to perturbations when friction has memory of normal stress history. The experiments of Prakash and Clifton (1993) and Prakash (1998) involve oblique shock impact of a hard metal plate against another made of a cutting tool material, so that slip at roughly constant velocity of order 1 to $10 \mathrm{~m} / \mathrm{s}$ begins at once. A reflected stress pulse is generated at the back face of the target plate which causes an abrupt decrease in normal stress that is presumably uniform all over the sliding surface. Laser-based optical interferometric measurement of the motion of the back face, on a time scale for which all waves can be regarded as one-dimensional, allows the interfacial slip rate and shear stress to be inferred.

The results suggest that there is no instantaneous change of shear strength, but rather a gradual change which occurs over a few microns of sliding. If analyzed with the regularizing friction law discussed by Martins and Simões (1995) and Simões and Martins (1998), in which the usual instantaneous dependence of shear strength on normal stress is replaced by a dependence on normal stress averaged over some small finite area, an abrupt decrease in shear strength would have been predicted (because the normal stress change was presumably uniform over the sliding surface). Such abrupt decrease was not observed. Instead, Prakash and Clifton (1993) and Prakash (1998) analyze their experiments in the framework of rate- and state-depen- 
dent friction, in which shear strength is regarded as a property of the current population of asperity contacts, and of their lifetimes, and it is only with ongoing slip or time that the population, and therefore the shear strength, can be altered. That is, there is no instantaneous dependence of shear strength on normal stress, but only an effect which has a fading memory dependence on recent normal stress history. In a constitutive law proposed by Prakash and Clifton (1993) and Prakash (1998), the strength is assumed to be altered by the slip but not directly by the time since a normal stress change, although such remains to be verified experimentally. Earlier studies by Linker and Dieterich (1992) at slip rates less than $1 \mu \mathrm{m} / \mathrm{s}$ imposed changes in normal stress during slip that were far less abrupt than those of the oblique shock experiments. Those workers found that there was a partial sudden change in the shear strength, followed by a gradual accumulation of the full effect of the altered normal stress over increasing slip and time. Since the shock experiments study the normal stress alteration over a shorter time scale, we believe that it is appropriate to use their result here, and to interpret the "sudden" change of shear strength of Linker and Dieterich (1992) as a feature of the slow creep slippage condition they studied, or of material differences (granite for them, versus hard metals against cutting tool materials in the shock experiments), or possibly as an artifact of their less abrupt change in time, which mapped memory effects into an apparent instantaneous effect. More experimental study of these issues is merited.

\subsection{A regularizing friction law}

We continue by using a simplification of the constitutive form suggested by Prakash and Clifton (1993) and Prakash (1998) which retains its essential feature for our purposes (regularization), namely, that there is a simple monotonic memory dependence but no instantaneous dependence of shear strength $\tau^{\mathrm{s}}$ on compressive normal stress $\sigma$. The form used is:

$$
\tau_{1}=\operatorname{sign}(V) \tau^{\mathrm{s}} \text { if } V \neq 0 ; \dot{\tau}^{\mathrm{s}}=-(|V| / L)\left(\tau^{\mathrm{s}}-f \sigma\right)
$$

where $\tau^{\mathrm{s}}>0,\left|\tau_{1}\right| \leq \tau^{\mathrm{s}}$ if $V=0$, and we are assuming $\sigma=-\tau_{2}>0 ; V$ is the sliding velocity and $L>0$ is a characteristic slip length over which the changes occur. No conclusion concerning regularization in the following development would change if we took $L$ proportional to $|V|$, so that evolution of strength with time, rather than with slip, if we simply replaced $|V| / L$ with an expression of form $a+b|V|>0$ where $a \geq 0$ and $b \geq 0$. We assume $V>0$ below so that Eq. (36) requires

$$
\dot{\tau}_{1}=-(V / L)\left(\tau_{1}+f \tau_{2}\right)
$$

Linearizing the above equation about the unperturbed slip rate (which amounts to treating $V / L$ as constant since its factor $\left(\tau_{1}+f \tau_{2}\right)$ already vanishes in the unperturbed state), taking Laplace transform, and considering a single Fourier mode as before, we get

$$
p \hat{T}_{1}=-(V / L)\left(\hat{T}_{1}+f \hat{T}_{2}\right)
$$

Using this relation in Eq. (22), imposing the constraint $\hat{D}_{2}=0$ and solving for $\hat{D}_{1}$, we get 


$$
\hat{D}_{1}=\frac{(p L / V+1)\left(\hat{K}_{11} \hat{K}_{22}-\hat{K}_{12} \hat{K}_{21}\right)}{(p L / V+1) \hat{K}_{22}-f \hat{K}_{21}} \hat{Q} .
$$

Thus, the equation governing stability is

$$
(s q+1) \hat{K}_{22}(s)-f \hat{K}_{21}(s)=0
$$

where $q=L|k| / V$.

We immediately see that no steady-state (i.e., $s$ pure imaginary) supersonic solutions of Eq. (40) can now exist for $k \neq 0$ since both $\hat{K}_{22}(s)$ and $\hat{K}_{21}(s)$ are purely imaginary in that region. In the long wavelength limit, $|k| \rightarrow 0$ and hence $q \longrightarrow 0$, the above equation reduces to Eq. (28), the governing equation for stability with constant Coulomb friction. Since ill-posedness relates to response as $|k| \rightarrow \infty$, it is of interest to know if there is stability at short wavelengths. This will require that the unstable roots of Eq. (28) move into left half plane or into a different Riemann sheet as $|k|$ is increased from 0 to $k_{\mathrm{cr}}$.

In the limit $|k| \rightarrow \infty$, equivalent to $q \rightarrow \infty$, the equation determining stability becomes $\hat{K}_{22}(s)=0$, which is precisely the condition for existence of the generalized Rayleigh wave in frictionless contact. Thus, if the material pair is sufficiently different that a generalized Rayleigh wave does not exist, which means that $\hat{K}_{22}(s)=0$ has no solution, then we are assured of stability at sufficiently large $|k|,|k|>k_{\mathrm{cr}}$. For cases where the generalized Rayleigh wave exists, a perturbation expansion in powers of $1 /|k|$ for the roots gives the root location at large $|k|$ as

$$
s=p /|k|=s_{0}+i s_{1} /|k|+\left(s_{2}+i s_{3}\right) / k^{2}+\cdots,
$$

where $s_{0}= \pm i c_{\mathrm{GR}}$ and $s_{1}, s_{2}$ and $s_{3}$ are real numbers with $s_{2}>0$ for one of the values of $s_{0}$. Therefore, a perturbation with large wavenumber $k$ grows as $\mathrm{e}^{s_{2} t / k \mid}$. This assures a finite integral over the amplitudes of all excited modes at all times and thus regularizes the problem. We thus see that for conditions under which the generalized Rayleigh wave exists in frictionless contact, all wavelengths are unstable with the friction law Eq. (36) as it was with the Coulomb friction law. However, the stability problem is now well-posed.

Generically, we find multiple bands of wavelengths that are unstable. We are concerned here only with determining the wavelength (or wavenumber $k_{\mathrm{cr}}$ ) at which the first unstable mode that causes the pathological ill-posedness is regularized. This mode is stabilized at short wavelengths when the material parameters are such that generalized Rayleigh wave does not exist in frictionless sliding. Particular cases are illustrated in Figs. 8-10. Fig. 8 is for a Steel/PMMA material pair. It was shown earlier in Fig. 5 that there are at most two unstable modes in the long wavelength limit when $f$ is between 0 and 2. The critical wavenumber $k_{\mathrm{cr}}$ above which the first unstable mode is stabilized is shown in Fig. 8.

Fig. 9 shows a similar plot for the case of a material pair with $\rho_{2} / \rho_{1}=1.2$ and $v_{1}=v_{2}=0.25$. We saw in Fig. 6 that the critical friction coefficient for this case is $f_{\mathrm{c}}=0.742$ when $c_{\mathrm{s} 2} / c_{\mathrm{s} 1}=1.7$ and $f_{\mathrm{c}}=0.184$ when $c_{\mathrm{s} 2} / c_{\mathrm{s} 1}=2.2$. Now, Fig. 9 shows the wavelength at which the first unstable mode is stabilized for these two cases. Fig. 10 is a similar plot for an interface with $\rho_{2} / \rho_{1}=0.8$ and $v_{1}=v_{2}=0.25$ at the same two values of $c_{\mathrm{s} 2} / c_{\mathrm{s} 1}$ as in the previous case. 


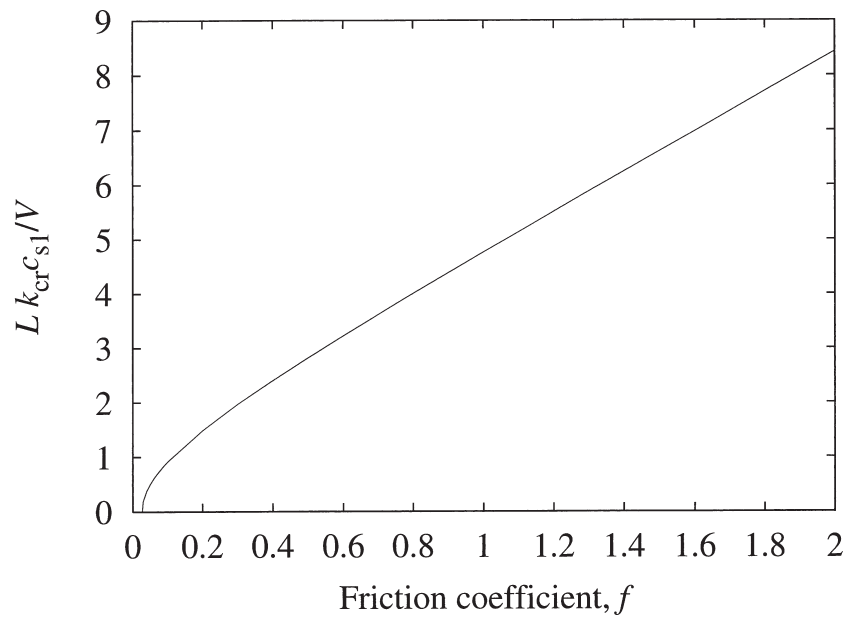

Fig. 8. Regularization of short wavelength instability with simplified Prakash-Clifton friction law for a Steel-PMMA material pair where $c_{\mathrm{s} 2} / c_{\mathrm{s} 1}=2.40, \rho_{2} / \rho_{1}=6.58, v_{1}=0.35$ and $v_{2}=0.3$.

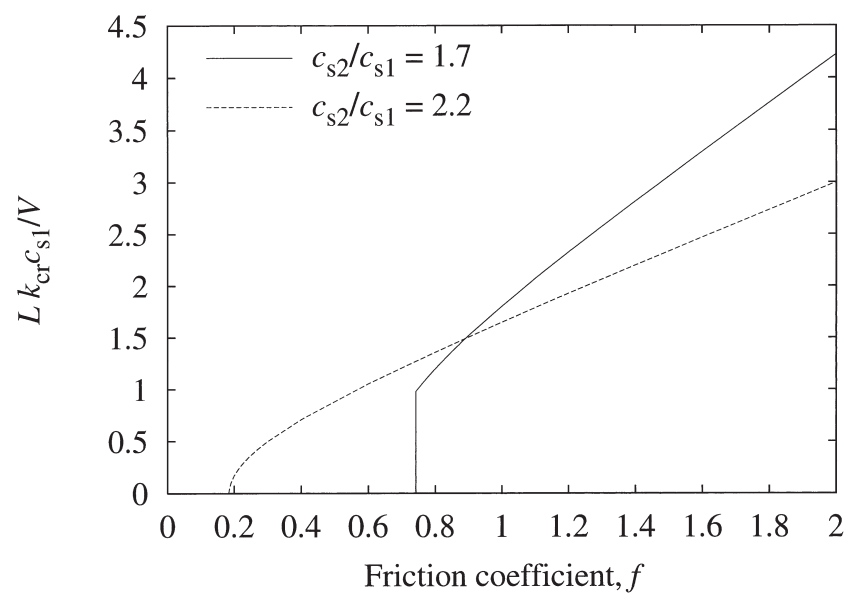

Fig. 9. Regularization of short wavelength instability with simplified Prakash-Clifton friction law for bi-material pairs with $\rho_{2} / \rho_{1}=1.2, v_{1}=v_{2}=0.25$.

\subsection{Further discussion of regularization}

As remarked, the existing experimental evidence (Prakash and Clifton (1993) and Prakash (1998)) at high slip rates of order 1 to $10 \mathrm{~m} / \mathrm{s}$, favors a memory-dependent description of friction in which the shear strength evolves continuously with slip and/or time in response to an instantaneous (step) change in compressive normal stress $\sigma$. If subsequent results validate, for the dynamic sliding range, the step change of shear strength with step of normal stress found by Linker and Dieterich (1992) 


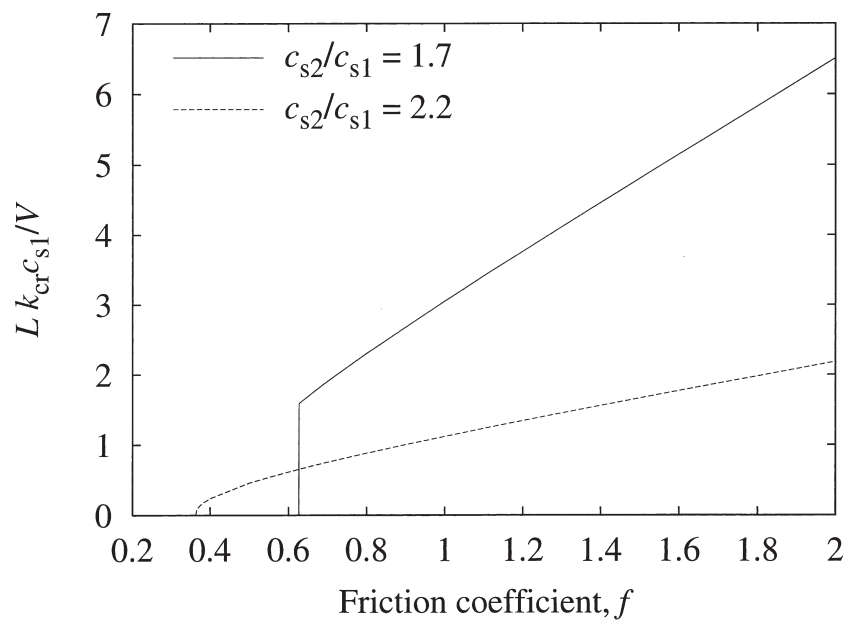

Fig. 10. Regularization of short wavelength instability with simplified Prakash-Clifton friction law for bi-material pairs with $\rho_{2} / \rho_{1}=0.8, v_{1}=v_{2}=0.25$.

for their creep-slippage experiments, then some other regularization would be required than that which we have discussed. We explain briefly how the regularization scheme of Martins and Simões (1995) and Simões and Martins (1998) can be analyzed in the present framework. For simplicity, we do this in the context of friction without any memory dependence so that, for slip situations which can be reduced to $2 \mathrm{D}$ perturbations like here, we write the shear strength as

$$
\tau^{\mathrm{s}}\left(x_{1}, t\right)=f \int_{x-D}^{x+D} w\left(\left|x_{1}^{\prime}-x_{1}\right|\right) \sigma\left(x_{1}^{\prime}, t\right) \mathrm{d} x_{1}^{\prime}
$$

where the weight $w\left(\left|x_{1}\right|\right) \geq 0$ and $\int_{-D}^{+D} w\left(\left|x_{1}^{\prime}\right|\right) \mathrm{d} x_{1}^{\prime}=1$. As before, $\tau_{1}=\tau^{\mathrm{s}}$ when $V>0$. Perturbations in modal shear and normal stresses of the form Eq. (4) at the interface are then related by

$$
T_{1}(t)=-\tilde{f}(k) T_{2}(t), \text { where } \tilde{f}(k)=f \int_{-D}^{+D} \cos \left(k x_{1}^{\prime}\right) w\left(\left|x_{1}^{\prime}\right|\right) \mathrm{d} x_{1}^{\prime} .
$$

Thus, the response to perturbation is the same problem as we have addressed in the body of the text, but with the classical Coulomb friction coefficient replaced by $\tilde{f}(k)$. Now, assuming that $w(|x|)$ is a function of bounded variation, we may assert on the basis of Fourier analysis that $|k \tilde{f}(k)|<B$ where $B$ is some bound valid for all $k$. Thus $\tilde{f}(k) \rightarrow 0$ as $|k| \rightarrow \infty$. 
Consider first cases for which the generalized Rayleigh wave does not exist. Then we showed that there is stability for sufficiently small $f$ (Figs. 5-7). As $|k|$ increases, $\tilde{f}(k)$ will fall into such a range. Hence the weighted friction law gives stable response at sufficiently large $|k|$ and regularizes such cases. When the generalized Rayleigh wave does exist, the sliding problem is unstable to perturbation for all non-zero $f$, but we showed (Eq. (33) and Eq. (34)) that when $f$ is changed slightly from zero, the real part of $s$ is proportional to $f$, and hence the growth (or decay) rate is of order $\pm k f$. But the corresponding quantity $\pm k \tilde{f}(k)$ is bounded as $|k| \rightarrow \infty$ for the weighted friction law. This implies that the growth rate is bounded, so that we retain instability as $|k| \rightarrow \infty$ but secure well-posedness.

\section{Summary}

The stability and well-posedness of steady frictional sliding along an interface between dissimilar linear elastic solids has been studied. It has been shown that with a Coulomb law, the stability problem is ill-posed for arbitrarily small friction when the solids on either side of the interface are such that the generalized Rayleigh wave exists in frictionless contact. Regularization of the problem by an experimentally motivated friction law incorporating the frictional response to normal stress changes has been demonstrated.

\section{Acknowledgements}

The study was supported by the Office of Naval research through grant N0001496-10777 to Harvard University, and through a Blaise Pascal Chair of the Foundation of Ecole Normale Superieure, Paris, to JRR. We are grateful to Drs. G. Adams and A. Cochard for discussions.

\section{References}

Achenbach, J.D., 1973. Wave propagation in elastic solids. North-Holland, Amsterdam.

Achenbach, J.D., Epstein, H.I., 1967. Dynamic interaction of a layer and a half-space. Journal of the Engineering Mechanics Division EM5, 27-42.

Adams, G.G., 1995. Self-excited oscillations of two elastic half-spaces sliding with a constant coefficient of friction. J. Appl. Mech. 62, 867-872.

Adams, G.G., 1998. Steady sliding of two elastic half-spaces with friction reduction due to interface stick-slip. J. Appl. Mech. 65, 470-475.

Adams, G.G., 2000. Radiation of body waves induced by the sliding of an elastic half-space against a rigid surface. J. Appl. Mech. 67, 105.

Andrews, D.J., Ben-Zion, Y., 1997. Wrinkle-like slip pulse on a fault between different materials. J. Geophys. Res. 102, 553-571.

Ben-Zion, Y., Andrews, D.J., 1998. Properties and implications of dynamic rupture along a material interface. Bull. Seismol. Soc. Amer. 88, 1085-1094. 
Breitenfeld, M.S., Geubelle, P.H., 1998. Numerical analysis of dynamic debonding under 2D in-plane and 3D loading. Int. J. Fracture 93, 13-38.

Cochard, A., Rice, J.R., 2001. Fault rupture between dissimilar materials: Ill-posedness, regularization, and slip-pulse response. Submitted to J. Geophys. Res.

Geubelle, P.H., Rice, J.R., 1995. A spectral method for three-dimensional elastodynamic fracture problems. J. Mech. Phys. Solids 43, 1791-1824.

Harris, R.A., Day, S.M., 1997. Effects of a low-velocity zone on a dynamic rupture. Bull. Seismol. Soc. Amer. 87, 1267-1280.

Linker, M.F., Dieterich, J.H., 1992. Effects of variable normal stress on rock friction: Observations and constitutive equations. J. Geophys. Res. 97, 4923-4940.

Martins, J.A.C., Guimaraes, J., Faria, L.O., 1995. Dynamic surface solutions in linear elasticity and viscoelasticity with frictional boundary conditions. Journal of Vibration and Acoustics, Trans. ASME 117, 445-451.

Martins, J.A.C., Simões, F.M.F., 1995. On some sources of instability/ill-posedness in elasticity problems with Coulomb friction. In: Raous, M., Jean, M., Moreau, J.J. (Eds.), Contact Mechanics. Plenum Press, New York, pp. 95-106.

Prakash, V., Clifton, R.J., 1993. Time resolved dynamic friction measurements in pressure-shear. In: Ramesh, K.T. (Ed.), Experimental Techniques in the Dynamics of Deformable Solids, AMD-vol. 165. ASME, New York, pp. 33-48.

Prakash, V., 1998. Frictional response of sliding interfaces subjected to time varying normal pressures. Journal of Tribology, Trans. ASME 120, 97-102.

Renardy, M., 1992. Ill-posedness at the boundary for elastic solids sliding under Coulomb friction. Journal of Elasticity 27, 281-287.

Simões, F.M.F., Martins, J.A.C., 1998. Instability and ill-posedness in some friction problems. Int. J. Eng. Sci. 36, 1265-1293.

Weertman, J., 1963. Dislocations moving uniformly on the interface between isotropic media of different elastic properties. J. Mech. Phys. Solids 11, 197-204.

Weertman, J., 1980. Unstable slippage across a fault that separates elastic media of different elastic constants. J. Geophys. Res. 85, 1455-1461. 